\title{
Meta-Analysis of Changes in Sleep Quality of Women with Breast Cancer before and after Therapy
}

\author{
Wen-Pei Chang ${ }^{a, b} \quad$ Yu-Pei Chang ${ }^{a}$ \\ ${ }^{a}$ Department of Nursing, Shuang Ho Hospital, Taipei Medical University, Ministry of Health and Welfare, \\ New Taipei City, Taiwan; ${ }^{b}$ School of Nursing, College of Nursing, Taipei Medical University, Taipei, Taiwan
}

\section{Keywords}

Women with breast cancer - Cancer therapy - Sleep quality · Meta-analysis

\begin{abstract}
Cancer treatments may affect the sleep quality and even future quality of life of women with breast cancer. A meta-analysis was performed to understand the changes in the sleep quality of women with breast cancer during their treatment period. In a systematic literature review in compliance with the PRISMA guidelines, we searched for articles published between 2000 and 2018 in databases. A total of 12 study articles were included. The standardized mean differences of the pooling effect size of sleep quality between the period before treatment and 1-8 weeks, 9-16 weeks, 17-24 weeks, and 25-56 weeks after the commencement of treatment were $-0.020,-0.162,0.075$, and 0.216 , respectively. Although the differences were not statistically significant, in view of the heterogeneity among the studies, we conducted further analysis using a linear mixed effect model. The overall results indicated poorer sleep quality as time passed from the start of the first treatment $(p=0.014)$. The results of this study revealed that patients experienced better sleep quality in the initial months after the beginning of treatment; however, their sleep quality became poorer between 4 months to ap-
\end{abstract}

proximately 1 year after the beginning of treatment, compared with the sleep quality before treatment, and continued to decline rather than improve during the follow-up period.

(c) 2019 S. Karger AG, Basel

\section{Introduction}

Sleep disorders are the most common and profoundly influencing symptom in cancer patients. Among the various types of cancer patients, breast cancer patients have the highest prevalence of sleep disturbance and voice the most subjective complaints, suggesting that breast cancer patients have a poorer sleep quality than do other cancer patients [1].

In terms of treatment, the National Comprehensive Cancer Network (NCCN) has listed four methods of treatment for breast cancer: surgery, radiation therapy, chemotherapy, and hormone therapy [2]. For women diagnosed with early-stage breast cancer, surgery is the initial treatment, which includes breast conserving surgery, modified radical mastectomy, and sentinel node biopsy [3]. Physicians then recommend radiation therapy in the 5-7 weeks following surgery to reduce the chance of recurrence [4]. In recent years, rapid progress has also been made in ra- 
diation therapy. For instance, accelerated partial breast irradiation can be used in patients with early-stage breast cancer and limits the range of irradiation to the area near the primary tumor, thereby reducing the treatment area as well as the number of treatments required. Moreover, accelerated partial breast irradiation is nearly as effective as conventional whole-breast radiotherapy but minimizes the amount of unnecessary irradiation to the heart and lungs, leading to a reduction in side effects [5]. In contrast, chemotherapy is administered for several weeks before or after surgery, especially in the event of metastases. Three types of adjuvant chemotherapy drugs are used after breast cancer surgery: the first-generation drug methotrexate, which is used less frequently at present; the second-generation drug adriamycin, which is effective in patients who have no lymph node metastasis; and the thirdgeneration drug is a mix of adriamycin and docetaxel or paclitaxel, which is mostly used to treat locally advanced breast cancer, early-stage breast cancer with lymph node metastasis, and triple-negative breast cancer with no lymph node metastasis [6]. Furthermore, hormone therapy to treat breast cancer, which is the cornerstone of treatment for advanced breast cancer, is equally as chemotherapy; however, hormonal drugs can only be used to inhibit the growth of breast cancer cells in patients with hormone receptor-positive breast cancer, i.e., ER+/HER2- (estrogen receptor-positive/human epidermal growth factor receptor 2-negative). Tamoxifen is the most common hormone medication for breast cancer treatment and can be administered before or after aromatase inhibitors, the former of which is typically prescribed for several years [7]. Targeted drugs, particularly those that are designed to target specific features of cancer cells, exclusively act on HER2 on the membranes of breast cancer cells and have become a newly emerging option for breast cancer treatment in recent years. Although these drugs cause fewer side effects than conventional chemotherapy does and have less impact on the quality of life during treatment, targeted drugs are not suitable for all breast cancer patients. Certain targeted drugs are only effective in patients whose breast biopsies exhibit HER2 overexpression [8].

In fact, half of the women with breast cancer complain of sleep disturbances at any point, starting from diagnosis to as late as 5 years after their cancer treatment had ended [9]. In other words, these disturbances not only appear during the pathological stage of breast cancer and the treatment period but are also problems that persist in the long term [10].

Recent studies have indicated that sleep disturbances in women with breast cancer affect many measurements that make up quality of life. Those with sleep deprivation perform more poorly at work and at everyday tasks, experience more physical pain, and have a poorer mental health [11]. The studies above demonstrate that the con- sequences of sleep disorders in cancer patients cover a wide range of aspects. We therefore performed a metaanalysis to understand the changes in the sleep quality of women with breast cancer during their treatment period.

\section{Materials and Methods}

\section{Empirical Literature Search Strategies}

To perform a systematic literature review, we searched for articles published in English between 2000 and 2018 in databases including PubMed, ProQuest, the Cumulative Index to Nursing and Allied Health Literature (CINAHL), and the Cochrane Library. Our screening process is shown in Figure 1. Our inclusion criteria for the collection and assessment of articles were female patients who had been diagnosed with breast cancer and had received cancer treatment. Our exclusion criteria were discourse or retrospective research articles and animal studies.

\section{Literature Quality}

The literature review protocol was developed in accordance with the PRISMA checklist. The articles remaining following the screening process were then reviewed using the appraisal tools formulated by the Joanna Briggs Institute (JBI). The appraisal criteria for nonrandomized experimental studies include whether the subjects were representative of patients in the population as a whole, whether the patients were at a similar point in the course of their condition/illness, whether bias had been minimized in relation to the selection of cases and controls, whether confounding factors were identified and strategies to deal with them stated, whether outcomes were assessed using objective criteria, whether follow-up was carried out over a sufficient time period, whether the outcomes of patients who withdrew were described and included in the analysis, whether outcomes were measured in a reliable way, and whether appropriate statistical analysis was used. Each article was given 1 point for each checklist item or 0 points if the article did not meet the criteria or if it was unclear whether it met the criteria [12]. In our analysis, only articles with a total score of 4 or more were included. The quality of each article was evaluated by two reviewers, and the consistency of their assessments was analyzed using the Kappa consistency coefficient.

\section{Data Analysis}

Data analysis was performed using Comprehensive MetaAnalysis (CMA) version 2.2.048 and SPSS version 19.0 (SPSS Inc., Chicago, IL, USA). Before combining the study articles and analyzing the results, we first tested the heterogeneity of the results of the studies using Cochran's Q test and presented the relative importance of the studies using a forest plot. Next, we checked whether eliminating any study influenced the overall results using sensitivity analysis and used a linear mixed effect model. The pretreatment period was designated as the time before radiation therapy, chemotherapy, hormone therapy, or targeted therapy. The time points designated after therapies had commenced were as follows: 1-8 weeks (Time 1), 9-16 weeks (Time 2), 17-24 weeks (Time 3), and 25-56 weeks (Time 4, the end of therapy).

\section{Results}

\section{Study Sample Attributes and Quality}

Using our search strategies, we ultimately obtained a total of 12 articles; the quality assessment scores of these 


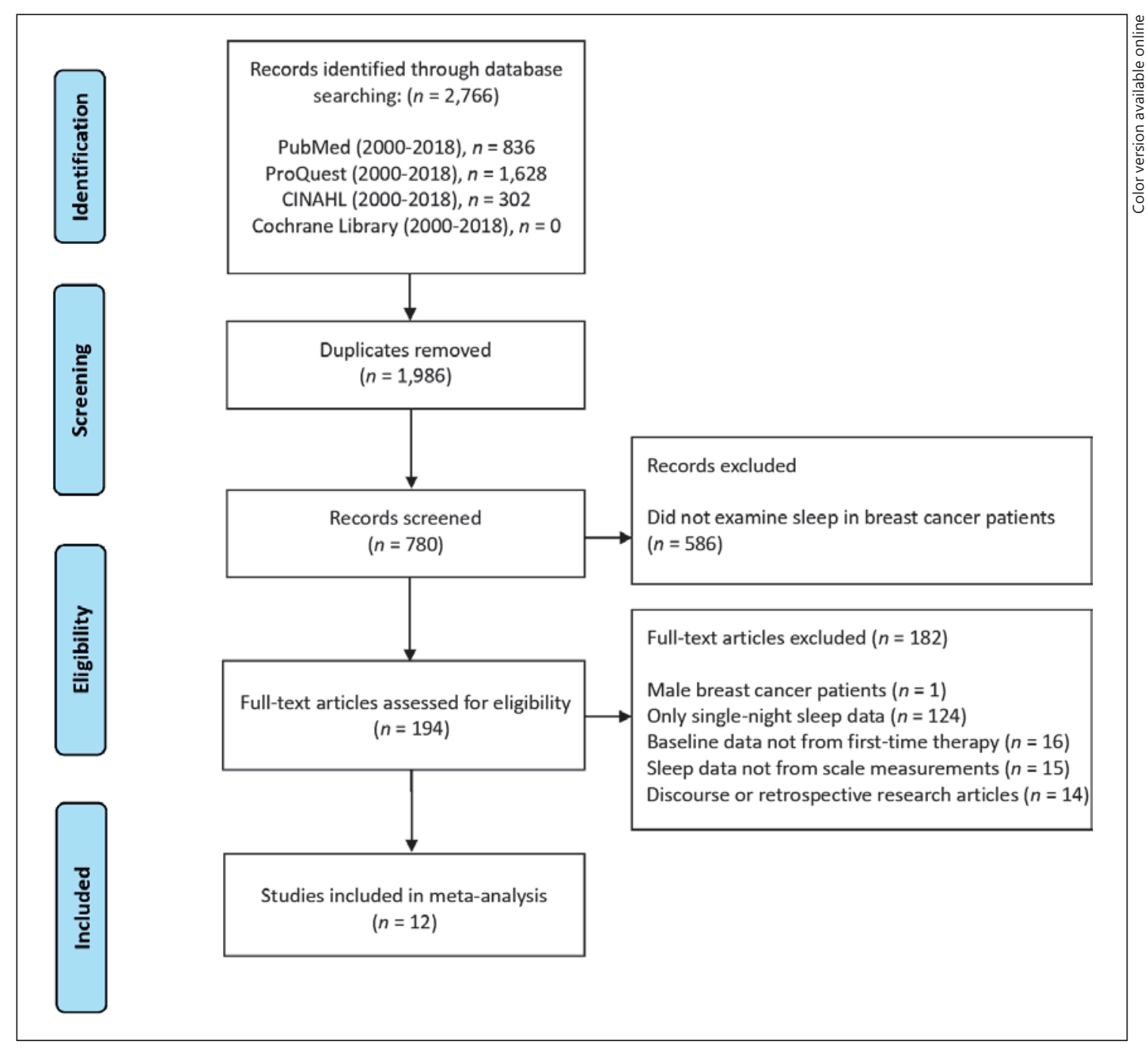

Fig. 1. PRISMA flow diagram for the study screening process.

fell between 6 and 8 points (Table 1). The Kappa coefficient of consistency between the quality assessments of the articles by the two reviewers was $0.871(p<0.001)$.

Heterogeneity Test, Pooling Effect Size, and Sensitivity Analysis of Baseline and Time 1

The heterogeneity test presented statistically significant differences $(p=0.020)$, with the percentage of variation due to heterogeneity $\left(I^{2}\right)$ being $55.87 \%$. This indicates a high degree of heterogeneity among the 7 studies; therefore, we chose the random effect model. The standardized mean difference (SMD) of the pooling effect size was -0.020 , whereas the $95 \%$ confidence interval was -0.146 to 0.106 , indicating that sleep quality at Time 1 was better than at baseline; however, the difference was not significant ( $p=0.752$ ) (Fig. 2). The elimination of any paper did not have a significant impact on the pooling effect size (95\% CI: $-0.146,0.106)$.

Sleep Quality of Women with Breast Cancer
Heterogeneity Test, Pooling Effect Size, and Sensitivity Analysis of Baseline and Time 2

The heterogeneity test presented statistically significant differences $(p<0.001)$, with the percentage of variation due to heterogeneity $\left(I^{2}\right)$ being $81.69 \%$. This indicates a high degree of heterogeneity among the 8 studies; therefore, we chose the random effect model. The SMD of the pooling effect size was -0.162 , and the $95 \%$ confidence interval was -0.359 to 0.036 , indicating that sleep quality at Time 2 was better than at baseline; however, the difference was not significant $(p=0.108)$ (Fig. 2). The elimination of any paper did not have a significant impact on the pooling effect size ( $95 \% \mathrm{CI}:-0.359,0.036)$.

Heterogeneity Test, Pooling Effect Size, and Sensitivity Analysis of Baseline and Time 3

The heterogeneity test presented statistically significant differences $(p<0.001)$, with the percentage of varia- 


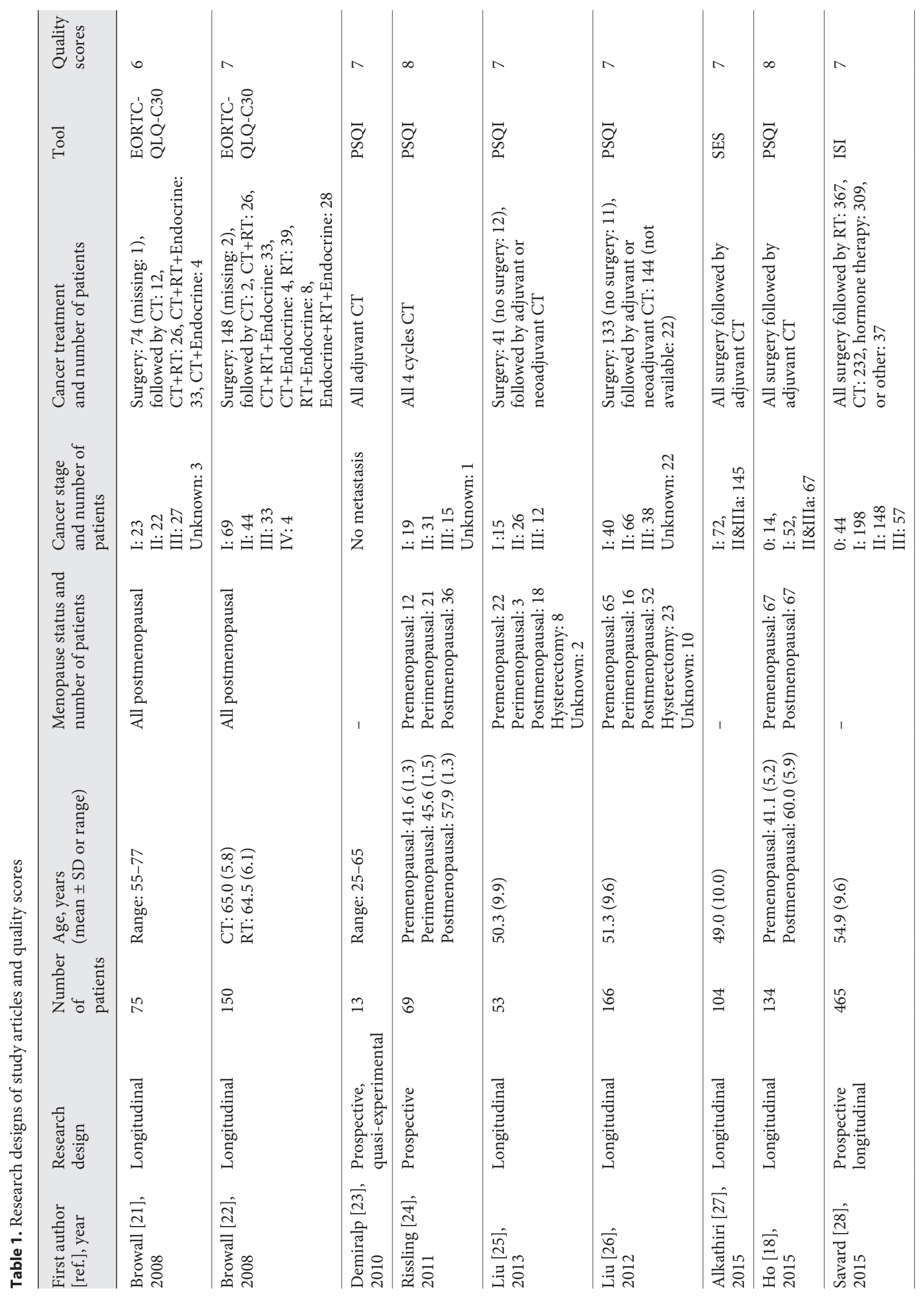




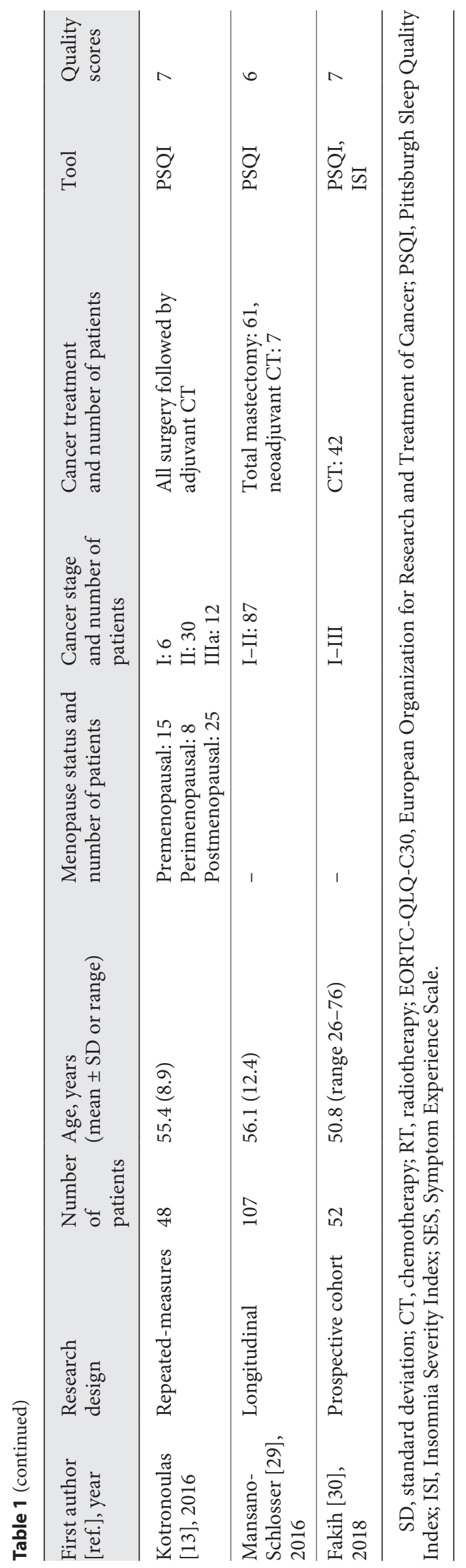

Sleep Quality of Women with Breast Cancer tion due to heterogeneity $\left(I^{2}\right)$ being $71.81 \%$, and thus indicating a high degree of heterogeneity among the 7 studies; therefore, the random effect model was chosen. The SMD of the pooling effect size was 0.075 , and the $95 \%$ confidence interval was -0.072 to 0.221 , meaning that sleep quality at Time 3 was poorer than at baseline; however, the difference was not significant ( $p=0.317$ ) (Fig. 2). The elimination of any paper did not have a significant impact on the pooling effect size (95\% CI: $-0.072,0.221)$.

Heterogeneity Test, Pooling Effect Size, and Sensitivity Analysis of Baseline and Time 4

The heterogeneity test presented statistically significant differences $(p<0.001)$, with the percentage of variation due to heterogeneity $\left(I^{2}\right)$ being $80.95 \%$. This indicates a high degree of heterogeneity among the 4 studies; therefore, the random effect model was chosen. The SMD of the pooling effect size was 0.216 , and the $95 \%$ confidence interval was -0.014 to 0.446 , indicating that sleep quality at Time 4 was poorer than at baseline; however, the difference was not significant ( $p=0.066$ ) (Fig. 2). The elimination of any paper did not have a significant impact on the pooling effect size ( $95 \% \mathrm{CI}:-0.014,0.446)$.

\section{Results of Pooling Effect Size and Linear Mixed Effect Model}

As shown above, despite the individual comparisons of the sleep quality at Time 1 , Time 2 , Time 3 , and Time 4 compared to the baseline, the SMD of the pooling effect size showed no significant differences (Fig. 3). Taking into consideration the heterogeneity among the studies, we conducted further analysis using a linear mixed effect model, the results of which indicated that the sleep quality of patients declined as the follow-up time increased $(p=0.014)($ Table 2$)$.

\section{Discussion and Conclusion}

The results of this study revealed that patients may have experienced better sleep quality in the initial months after the beginning of treatment compared with the sleep quality of the patients before treatment; however, between 4 months to approximately 1 year following the commencement of treatment, their sleep quality became poorer compared to the period prior to treatment. Nonetheless, our linear mixed effect model indicates that during cancer treatment, the sleep disorders of women with breast cancer were exacerbated as the treatment time increased and were significantly more severe than before undergoing treatment.

Aside from the sleeping difficulty score in the qualityof-life questionnaire from the European Organization for Research and Treatment of Cancer (EORTC)-QLQ-C30, 


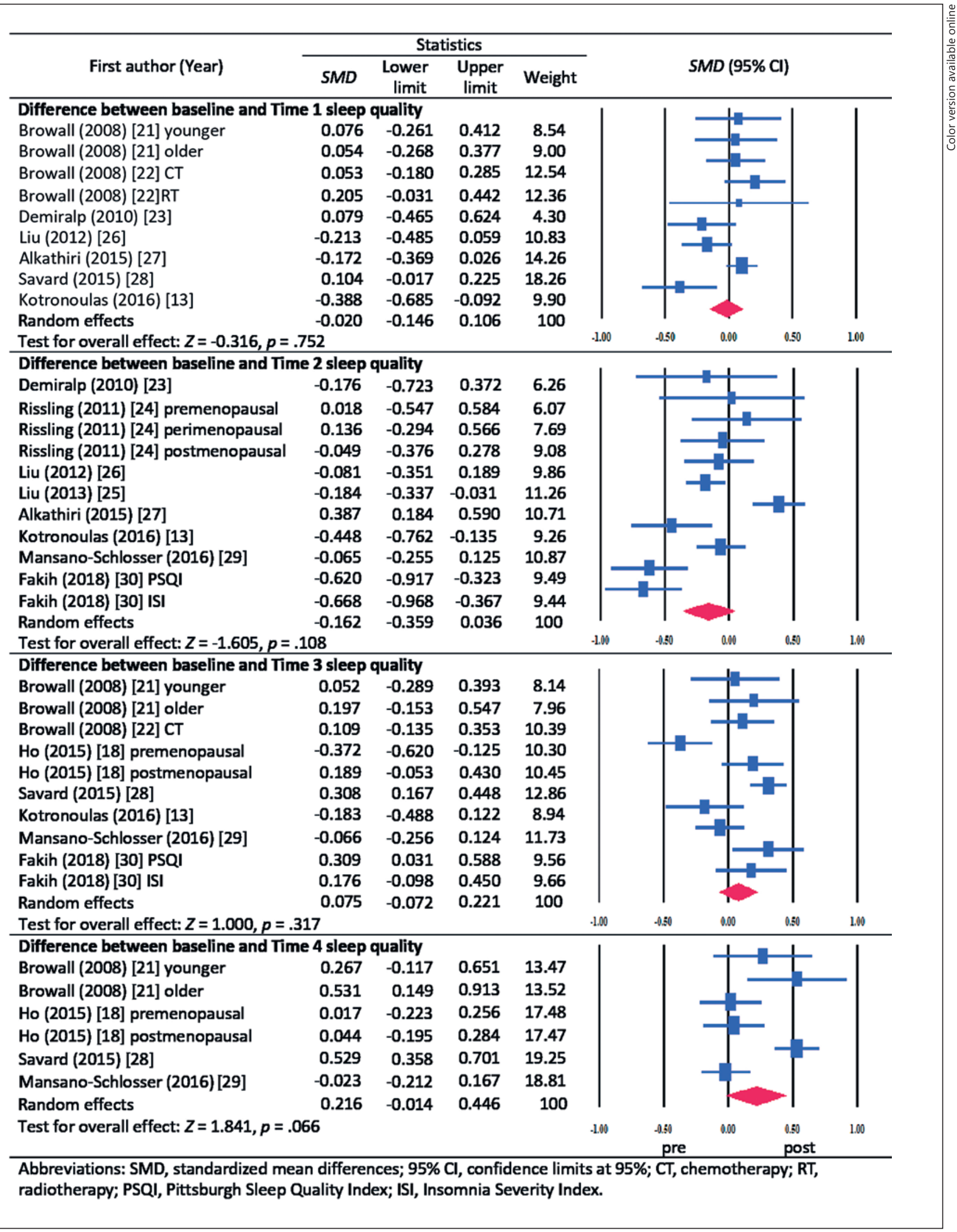

Fig. 2. Forest plots of differences in the sleep quality before and after therapy. 
Fig. 3. Summary estimates of effect with all observations.

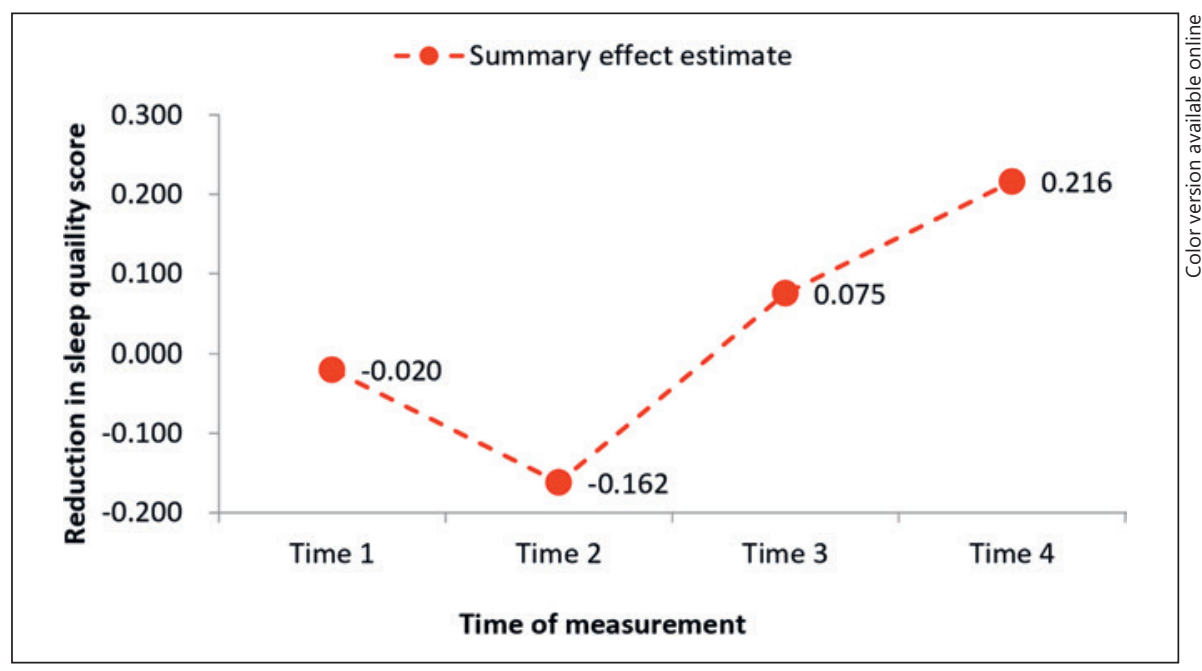

Table 2. Weighted linear mixed model

\begin{tabular}{|c|c|c|c|c|c|}
\hline \multirow[t]{2}{*}{ Variable } & \multirow[t]{2}{*}{ Estimate } & \multirow[t]{2}{*}{ SE } & \multirow[t]{2}{*}{$p$ value } & \multicolumn{2}{|l|}{$95 \% \mathrm{CI}$} \\
\hline & & & & lower & upper \\
\hline Intercept & -0.244 & 0.105 & 0.026 & -0.457 & -0.031 \\
\hline Follow-up time (Time $1,2,3,4$ ) & 0.102 & 0.039 & 0.014 & 0.022 & 0.182 \\
\hline
\end{tabular}

SE, standard error; $95 \% \mathrm{CI}$, confidence limits at 95\%.

the Insomnia Severity Index (ISI), and the Symptom Experience Scale (SES), which were used in four studies, most of the studies employed the Pittsburgh Sleep Quality Index (PSQI) to assess the sleep quality of patients, which is also clinically and frequently used to evaluate the sleep quality of cancer patients [13]. Many reports have indicated that the primary sleep problems of women with breast cancer include difficulty falling asleep, waking in the middle of the night, difficulty waking up, and excessive daytime sleepiness [14]. Fontes et al. [15] tested the reliability and validity of PSQI scores from 474 breast cancer patients and found good internal consistency and construct validity (Cronbach's $\alpha=0.7$ ). A cumulative score of $\leq 5$ indicates substantially good sleep quality. Following a suspected presence of breast cancer, patients must undergo a series of tests or surgeries to confirm the pathological diagnosis before receiving treatment. Their worries about the side effects of various treatments, and the discomforts brought on by their cancer or the treatments may interrupt their daily routines, all of which could affect the quality of their sleep [16]. Similarly, the results of this study indicate that women with breast cancer already have sleep problems before they receive treatment. Most patients had higher PSQI scores before receiving treatment; therefore, the sleep problems may be worse before treatment than during the first months of treatment.

Sleep Quality of Women with Breast Cancer
Sleep problems in women with breast cancer undergoing postsurgical therapy were strongly linked to estrogen depletion and deteriorating physiological functions [17]. Women with breast cancer who received chemotherapy around the time of menopause often displayed severe sleep disorders, depression, and fatigue [18]. Although the above studies reported profound consequences on sleep quality after cancer treatment, none examined sleep conditions before treatment and therefore could not perform comparisons with the baseline sleep quality before treatment. The results of the present study indicate that women with breast cancer already have sleep problems before they receive the abovementioned treatments.

Although the 5-year survival rate of cancer has greatly improved, breast cancer patients still face different physical symptoms during the remission and survival periods after treatment, and the presence of sleep problems may affect the overall quality of their lives [19]. Fleming et al. [20] interviewed a focus group of 21 cancer survivors regarding their sleep problems. They found that sleep problems are widely prevalent among cancer survivors and have an impact on emotional, physiological, behavioral, interpersonal, and cognitive levels. Emotionally, they may display irritability, frustration, and helplessness; physiologically, they may experience fatigue and headaches; they may often nap during the day and stay in bed. 
All of these changes only further aggravate sleep problems. Our meta-analysis also confirmed that the sleep quality 4 months to approximately 1 year after the beginning of therapy is indeed poorer than the sleep quality before therapy started.

\section{Study Limitation}

As this study only compiled the results of a small number of sleep-related scales, the objectivity in the results obtained may be limited. We do not know whether the conclusions regarding changes in sleep quality would be consistent with those of other tools, such as Actigraphy. Thus, we suggest that future investigations compare various sleep quality assessment tools to gain further understanding of the changes in the sleep quality during the treatment period of breast cancer patients.

\section{Conclusion}

The results of this study indicate that an association exists between sleep quality and therapy after surgery in breast cancer patients. While interventions may be able to relieve the uncomfortable side effects of cancer treatment and improve the sleep quality of patients during their treatment, our results indicate that even when breast cancer patients begin their follow-up period after treatment, their sleep problems worsen instead of improving.

\section{Acknowledgement}

We thank the anonymous reviewers and editor for their comments.

\section{Statement of Ethics}

The authors have no ethical conflicts to disclose.

\section{Disclosure Statement}

The authors have no conflicts of interest to disclose.

\section{Funding Sources}

Research reported in this publication was supported by the Taipei Medical University-Shuang Ho Hospital, Ministry of Health and Welfare, under the award No. 108HCP-11.

\section{Author Contributions}

Wen-Pei Chang conceived of the presented idea, developed the theory, and performed the computations. Wen-Pei Chang and $\mathrm{Yu}$ Pei Chang verified the analytical methods. Wen-Pei Chang supervised the findings of this work. All authors discussed the results and contributed to the final manuscript.

\section{References}

1 Palesh OG, Collie K, Batiuchok D, Tilston J, Koopman C, Perlis ML, et al. A longitudinal study of depression, pain, and stress as predictors of sleep disturbance among women with metastatic breast cancer. Biol Psychol. 2007 Apr;75(1):37-44

2 Gradishar WJ, Anderson BO, Balassanian R, Blair SL, Burstein HJ, Cyr A, et al. Breast cancer, Version 4.2017, NCCN clinical practice guidelines in oncology. J Natl Compr Canc Netw. 2018 Mar;16(3):310-20.

3 Zahoor S, Haji A, Battoo A, Qurieshi M, Mir W, Shah M. Sentinel lymph node biopsy in breast cancer: a clinical review and update. J Breast Cancer. 2017 Sep;20(3):217-27.

4 Karlsson P, Cole BF, Price KN, Gelber RD, Coates AS, Goldhirsch A, et al.; International Breast Cancer Study Group. Timing of radiation therapy and chemotherapy after breast-conserving surgery for node-positive breast cancer: long-term results from International Breast Cancer Study Group Trials VI and VII. Int J Radiat Oncol Biol Phys. 2016 Oct;96(2):273-9.

5 Tann AW, Hatch SS, Joyner MM, Wiederhold LR, Swanson TA. Accelerated partial breast irradiation: Past, present, and future. World J Clin Oncol. 2016 Oct;7(5):370-9.

6 Kumar P, Aggarwal R. An overview of triplenegative breast cancer. Arch Gynecol Obstet. 2016 Feb;293(2):247-69.
7 Runnak MA, Hazha MA, Hemin HA, Wasan AA, Rekawt RM, Michael HD. A populationbased study of Kurdish breast cancer in northern Iraq: hormone receptor and HER2 status. A comparison with Arabic women and United States SEER data. BMC Womens Health. 2012 Jun;12(1):16.

8 Larionov AA. Current therapies for human epidermal growth factor receptor 2-positive metastatic breast cancer patients. Front Oncol. 2018 Apr;8:89.

9 Sanford SD, Wagner LI, Beaumont JL, Butt Z, Sweet JJ, Cella D. Longitudinal prospective assessment of sleep quality: before, during, and after adjuvant chemotherapy for breast cancer. Support Care Cancer. 2013 Apr;21(4):959-67.

10 İzci F, İlgün AS, Fındıklı E, Özmen V. Psychiatric symptoms and psychosocial problems in patients with breast cancer. J Breast Health. 2016 Jul;12(3):94-101.

11 Rogers LQ, Courneya KS, Oster RA, Anton PM, Robbs RS, Forero A, et al. Physical activity and sleep quality in breast cancer survivors: a randomized trial. Med Sci Sports Exerc. 2017 Oct;49(10):2009-15.

12 Joanna Briggs Institute. Joanna Briggs Institute reviewers' manual. Australia: University of Adelaide; 2011.

13 Kotronoulas G, Wengström Y, Kearney N. Alterations and interdependence in self- reported sleep-wake parameters of patientcaregiver dyads during adjuvant chemotherapy for breast cancer. Oncol Nurs Forum. 2016 May;43(3):288-301.

14 Trudel-Fitzgerald C, Zhou ES, Poole EM, Zhang X, Michels KB, Eliassen AH, et al. Sleep and survival among women with breast cancer: 30 years of follow-up within the Nurses' Health Study. Br J Cancer. 2017 Apr;116(9): 1239-46.

15 Fontes F, Gonçalves M, Maia S, Pereira S, Severo M, Lunet N. Reliability and validity of the Pittsburgh Sleep Quality Index in breast cancer patients. Support Care Cancer. 2017 Oct;25(10):3059-66.

16 Moore HC. Breast cancer and sleep disturbance: more than simply a quality of life concern. Ann Palliat Med. 2012 Oct;1(3): 211-2.

17 Fiorentino L, Rissling M, Liu L, Ancoli-Israel S. The symptom cluster of sleep, fatigue and depressive symptoms in breast cancer patients: severity of the problem and treatment options. Drug Discov Today Dis Models. 2011;8(4):167-73.

18 Ho SY, Rohan KJ, Parent J, Tager FA, McKinley PS. A longitudinal study of depression, fatigue, and sleep disturbances as a symptom cluster in women with breast cancer. J Pain Symptom Manage. 2015 Apr;49(4):707-15. 
19 Whisenant M, Wong B, Mitchell SA, Beck SL, Mooney K. Distinct trajectories of fatigue and sleep disturbance in women receiving chemotherapy for breast cancer. Oncol Nurs Forum. 2017 Nov;44(6):739-50.

20 Fleming L, Gillespie S, Espie CA. The development and impact of insomnia on cancer survivors: a qualitative analysis. Psychooncology. 2010 Sep;19(9):991-6.

21 Browall MM, Ahlberg KM, Persson LO, Karlsson PO, Danielson EB. The impact of age on Health-Related Quality of Life (HRQoL) and symptoms among postmenopausal women with breast cancer receiving adjuvant chemotherapy. Acta Oncol. 2008;47(2):207-15.

22 Browall M, Ahlberg K, Karlsson P, Danielson E, Persson LO, Gaston-Johansson F. Healthrelated quality of life during adjuvant treatment for breast cancer among postmenopausal women. Eur J Oncol Nurs. 2008 Jul; 12(3):180-9.
23 Demiralp M, Oflaz F, Komurcu S. Effects of relaxation training on sleep quality and fatigue in patients with breast cancer undergoing adjuvant chemotherapy. J Clin Nurs. 2010 Apr;19(7-8):1073-83.

24 Rissling MB, Liu L, Natarajan L, He F, AncoliIsrael S. Relationship of menopausal status and climacteric symptoms to sleep in women undergoing chemotherapy. Support Care Cancer. 2011 Aug;19(8):1107-15.

25 Liu L, Fiorentino L, Rissling M, Natarajan L, Parker BA, Dimsdale JE, et al. Decreased health-related quality of life in women with breast cancer is associated with poor sleep. Behav Sleep Med. 2013;11(3):189-206.

26 Liu L, Mills PJ, Rissling M, Fiorentino L, Natarajan L, Dimsdale JE, et al. Fatigue and sleep quality are associated with changes in inflammatory markers in breast cancer patients undergoing chemotherapy. Brain Behav Immun. 2012 Jul;26(5):706-13.
27 Alkathiri AM, Albothi GK. Symptom cluster and severity among women with breast cancer undergoing chemotherapy in Saudi Arabia. J Med Sci. 2015 Feb;6(2):40-06.

28 Savard J, Ivers H, Savard MH, Morin CM. Cancer treatments and their side effects are associated with aggravation of insomnia: results of a longitudinal study. Cancer. 2015 May;121(10):1703-11.

29 Mansano-Schlosser TC, Ceolim MF. Longitudinal variations of sleep quality in women with breast cancer. Acta Paul Enferm. 2016 Oct;29(5):595-602.

30 Fakih R, Rahal M, Hilal L, Hamieh L, Dany M, Karam S, et al. Prevalence and severity of sleep disturbances among patients with early breast cancer. Indian J Palliat Care. 2018 Jan-Mar; 24(1):35-8. 